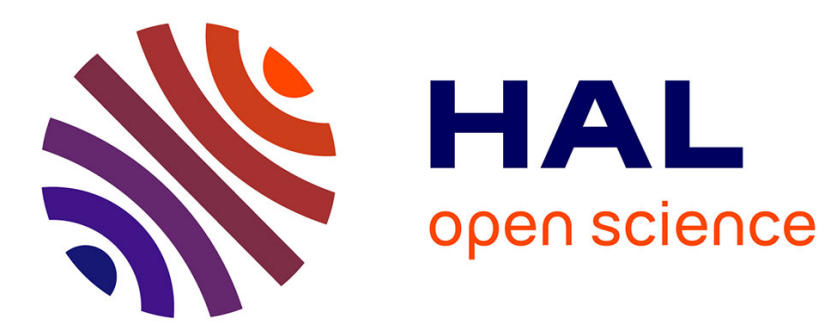

\title{
Novel approach to isoindolo[2,1-a]quinolines
}

Pascal Pigeon, Bernard Decroix

\section{To cite this version:}

Pascal Pigeon, Bernard Decroix. Novel approach to isoindolo[2,1-a]quinolines. Synthetic Communications, 1998, 28 (13), pp.2507-2516. 10.1080/00397919808004302 . hal-01230413

\section{HAL Id: hal-01230413 \\ https://hal.science/hal-01230413}

Submitted on 15 Feb 2018

HAL is a multi-disciplinary open access archive for the deposit and dissemination of scientific research documents, whether they are published or not. The documents may come from teaching and research institutions in France or abroad, or from public or private research centers.
L'archive ouverte pluridisciplinaire HAL, est destinée au dépôt et à la diffusion de documents scientifiques de niveau recherche, publiés ou non, émanant des établissements d'enseignement et de recherche français ou étrangers, des laboratoires publics ou privés. 


\title{
NOVEL APPROACH TO ISOINDOLO[2,1-a]QUINOLINES
}

\author{
Pascal Pigeon and Bernard Decroix ${ }^{*}$
}

Laboratoire de Chimie, Université du Havre, 30, rue Gabriel Péri, 76600 Le Havre, France

* To whom correspondence should be addressed.

\begin{abstract}
An $N$-acyliminium ion approach towards the synthesis of isoindolo [2,1-a] quinolines by an intramolecular process is described.
\end{abstract}

During the course of our work concerning the synthesis of new fused polyheterocyclic compounds with potential therapeutic interest we described in previous papers the use of an $N$-acyliminium ion in the key cyclization $\operatorname{step}^{1-3}$. We wish to report herein a novel approach to isoindolo[2,1-a]quinolines through an intramolecular cyclization using $\mathrm{N}$-acyliminium ion tethered to o-vinylbenzene derivatives. Few examples of the use of substituted vinylbenzenes as the $\pi$-nucleophile reagent are reported ${ }^{4-5}$ in the literature, probably due to the possible polymerization reaction in an acidic medium.

Various approaches ${ }^{6-8}$ leading to isoindolo[2,1-a]quinoline derivatives are described but to our knowledge none use an $\mathrm{N}$-acyliminium ion generated from an isoindole moiety. Thus we propose (Scheme 1) a novel approach to this tetracyclic system starting from suitable osubstituted aniline. To these compounds addition of a Grignard reagent (methylmagnesium iodide) or reduction $\left(\mathrm{NaBH}_{4}, \mathrm{CH}_{3} \mathrm{OH}\right)$ gave the corresponding alcohols 1a-c (>95\%). Treatment of 1a,b by $p$-toluenesulfonic acid (PTSA) afforded the vinyl derivatives 2a,b which directly reacted (one pot) with phthalic anhydride in the presence of triethylamine to furnish the phthalimides 3a,b (>90\%). Complete polymerization occurred during the acidic treatment of 1e. According to our preceding work $^{1}$, reduction of $\mathbf{3 a , b}$ using sodium borohydride or addition of a Grignard reagent led to the hydroxylactams $\mathbf{4 a}, \mathbf{b}\left(\mathrm{R}_{2}=\mathrm{H},>95 \%\right)$ and $\mathbf{5 a}, \mathbf{b}\left(\mathrm{R}_{2}=\mathrm{Me}\right)$ respectively. The latter were very sensitive to dehydration since during work up, formation of the enamides $\mathbf{6 a , b}$ (>95\% for $\mathbf{5 + 6}$ ) was observed. 


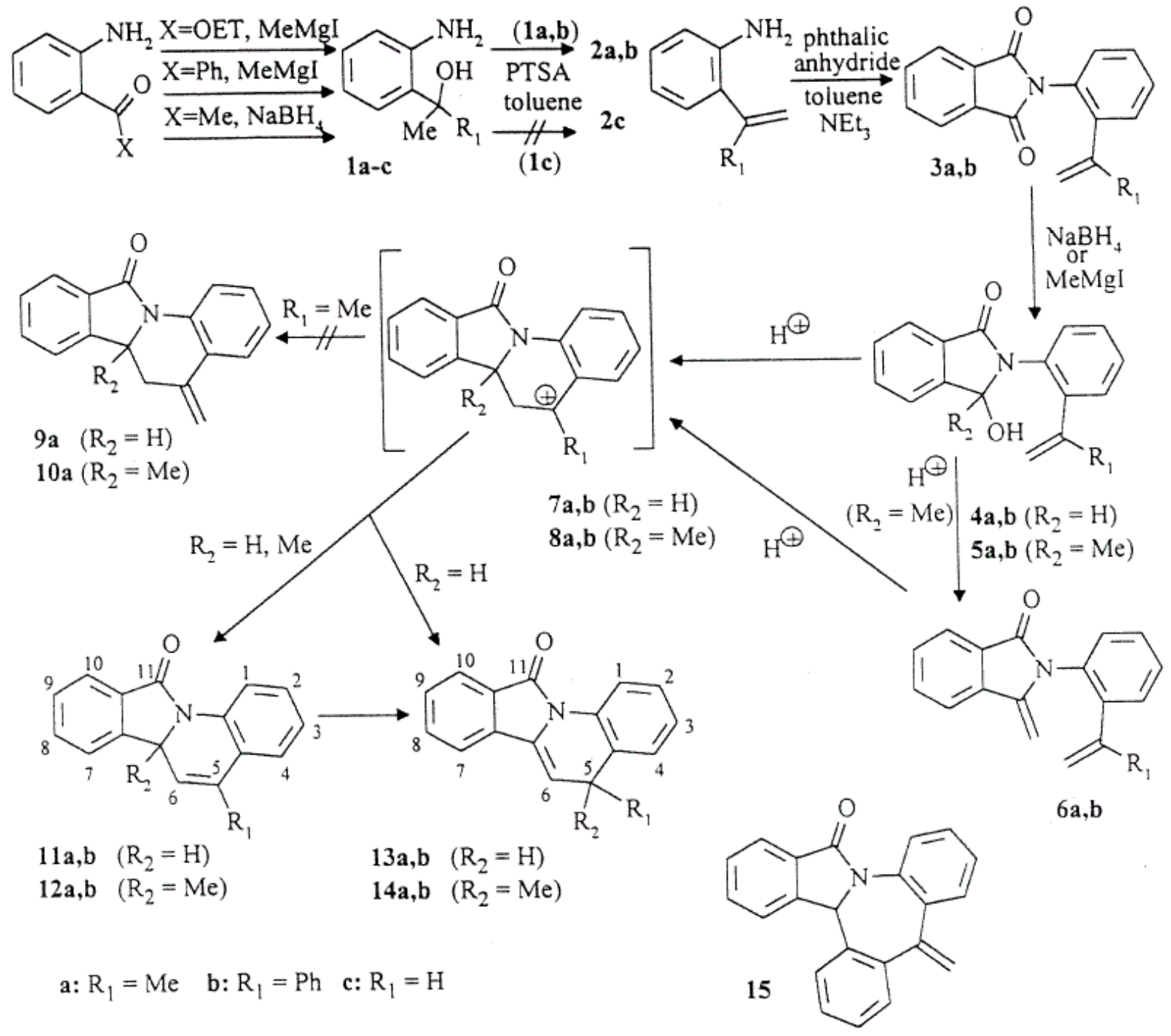

\section{Scheme 1}

Submitted to PTSA in refluxing toluene with heteroazeotropic elimination of water the hydroxylactams $\mathbf{4 a , b}$ (reaction time 30 minutes) or 5a,b (reaction time 4 hours) gave compounds 13a (93\%), 13b $(65 \%$, contaminated with $\mathbf{1 5})$ or $\mathbf{1 2 a}, \mathbf{b}(>90 \%)$ respectively. To explain the selectivity observed during the formation of these quinolines $\mathbf{1 2}$ or $\mathbf{1 3}$ we can consider the carbocations $\mathbf{7 a , b}$ and $\mathbf{8 a , b}$ which would result from a $\pi$-nucleophilic attack of the vinylbenzene onto the $N$-acyliminium ion generated from the hydroxylactams 4 or $\mathbf{5}$. In the case of 7a,b $\left(\mathrm{R}_{2}=\mathrm{H}\right)$ regardless of $\mathrm{R}_{1}\left(\mathrm{CH}_{3}\right.$, phenyl), elimination of the $\alpha$ proton led to the intermediate 11a,b which was rapidly isomerized to the stable enamides 13a,b. The possible compounds 9a, 10a and 11a,b were not detected in the reaction mixture. Nevertheless, a reaction conducted with trifluoroacetic acid in dichloromethane at room temperature gave 11a,b which transformed with time into the corresponding enamides $\mathbf{1 3 a}, \mathbf{b}(>90 \%$, in this case $\mathbf{1 3 b}$ was not contaminated with $\mathbf{1 5}$ ). We have already mentioned that the formation of a 
carbon-carbon double bond conjugated to the lactam function was very easy ${ }^{9,10}$. Furthermore, compounds 14a,b (consecutive to a 1,3 migration of a methyl group) have never been observed.

During the treatment of $\mathbf{4 b}$, another compound (15) was detected. It may result from the competitive $\pi$-aromatic attack of the benzene ring $\left(\mathrm{R}_{1}=\right.$ phenyl) upon the $N$-acyliminium ion to afford an isoindolo[a]dibenz[c,f]azepine analogous to the isoindolo[a]dibenz[c,e]azepine recently described by us $^{2}$. To confirm this observation, we undertook the synthesis of $\mathbf{1 8}$ (Scheme 2). The starting phthalimide 16 resulted from the condensation of phthalic anhydride with o-aminobenzophenone (93\%). Reduction of the ketone group (triethylsilane, TFA) followed by a reduction of the imide function $\left(\mathrm{NaBH}_{4}, \mathrm{CH}_{3} \mathrm{OH}\right)$ led to the hydroxylactam 17 (96\%). This latter species with trifluoroacetic acid at room temperature furnished the expected isoindolo[a]dibenz[c,f]azepine 18 (95\%). This excellent yield supports the hypothesis that in $\mathbf{4 b}$, the $\pi$-aromatic nucleophile can compete with the $\pi$-olefinic nucleophile during the attack of the $N$-acyliminium ion.

The structures of 12a,b, 13a,b, 18 were supported by their ir and nmr spectra as well as their microanalyses.

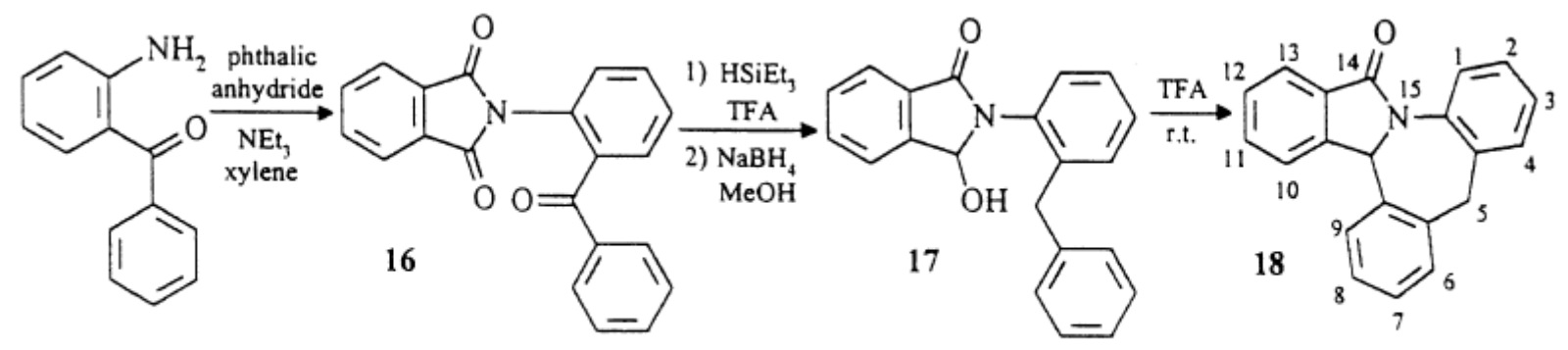

Scheme 2

Since the isoindolo[a]quinolines 11c or 13c $\left(R_{1}=R_{2}=H\right)$ could not be obtained in a similar manner, two other routes have been investigated (Scheme 3). Reaction of 1c with phthalic anhydride gave 19 (40\%) which was successively dehydrated (PTSA, toluene) into 3c (65\%) then reduced $\left(\mathrm{NaBH}_{4}, \mathrm{CH}_{3} \mathrm{OH}\right)$ to $\mathbf{4} \mathbf{c}\left(\mathrm{R}_{1}=\mathrm{R}_{2}=\mathrm{H}\right)(92 \%)$. Unfortunately, and as it could be expected this latter compound under acidic conditions (PTSA, toluene or TFA, $\mathrm{CH}_{2} \mathrm{Cl}_{2}$ ) gave rapid polymerization. Nevertheless alcohol $\mathbf{2 0}^{6}$ treated with PTSA in toluene with heteroazeotropic elimination of water gave 13c in an excellent yield (99\%). This compound has been previously prepared from a quinoline derivative ${ }^{7}$. The possible intermediate 11c has not been detected and the dehydration was very fast (10 minutes). 


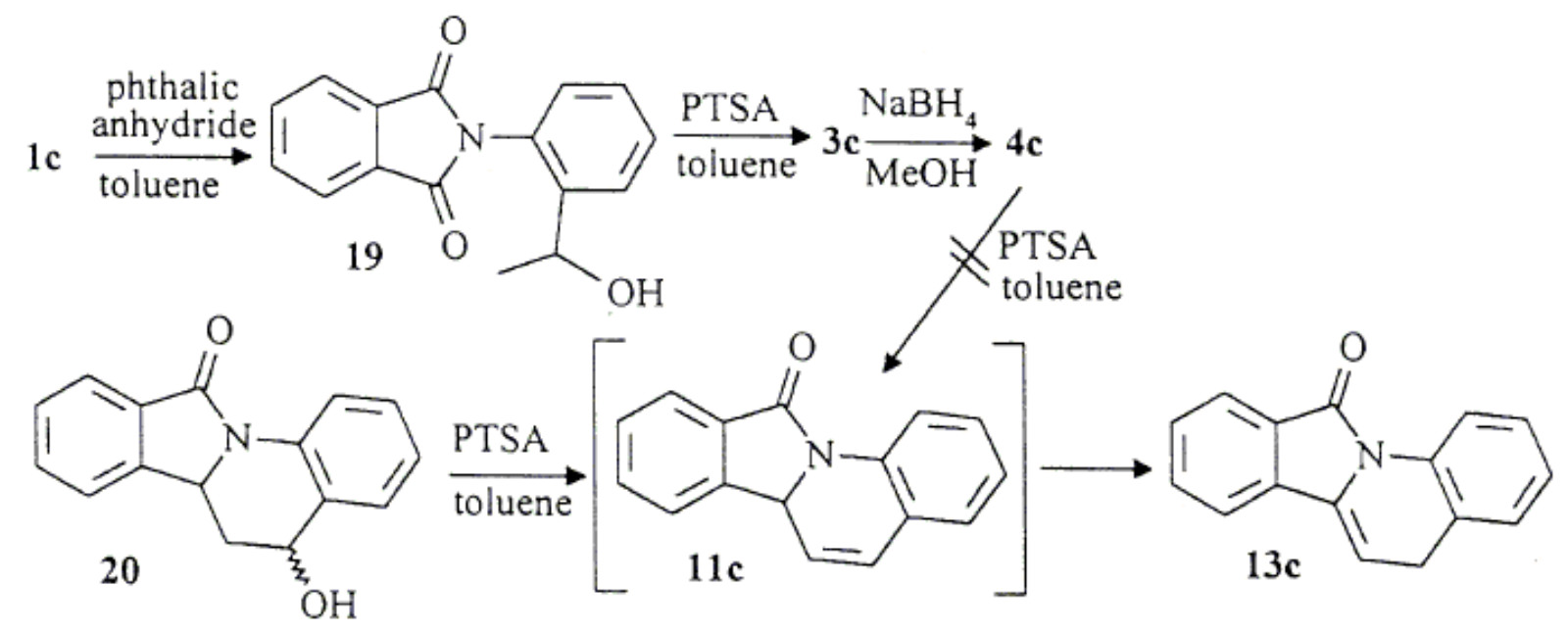

Scheme 3

In conclusion, we have demonstrated that substituted vinylbenzenes can be used as precursors for a $\pi$-nucleophilic attack onto $N$-acyliminium ion in an intramolecular process. This reaction constitutes a novel approach to the isoindolo[a]quinoline system and applications of this cyclization reaction to the synthesis of heterocyclic analogs are under investigations.

\section{EXPERIMENTAL}

Melting points are uncorrected. The infrared spectra of solids (potassium bromide) were recorded on a Perkin Elmer FTIR paragon 1000 spectrometer. The ${ }^{1} \mathrm{H}$ and ${ }^{13} \mathrm{C}$ NMR spectra were recorded on a Bruker AC-200 (200 MHz) instrument in deuteriochloroform solution and chemical shift $(\delta)$ are expressed in ppm relative to internal TMS. Ascending thin layer chromatography was performed on precoated plates of silica gel $60 \mathrm{~F} 254$ (Merck) and the spots visualized using an ultraviolet lamp or iodine vapor. E. Merck silica gel 60 F (70-300 mesh) was used for column chromatography. The elemental analyses were carried out by the microanalysis laboratory of INSA at Rouen, F $76130 \mathrm{M}^{\mathrm{t}}$. S $\mathrm{S}^{\mathrm{t}}$. Aignan, France.

\section{Synthesis of phthalimides 3a,b}

To a $0.5 \mathrm{M}$ solution of methylmagnesium iodide $(120 \mathrm{~mL}$ for $\mathbf{3 a}, 80 \mathrm{~mL}$ for $\mathbf{3 b})$ was added dropwise, with stirring, a solution of $10 \mathrm{mmol}$ of the corresponding amine (1.65 $\mathrm{g}$ of ethyl oaminobenzoate for $\mathbf{3 a}, 1.97 \mathrm{~g}$ of o-aminobenzophenone for $\mathbf{3 b}$ ) in $10 \mathrm{~mL}$ of dry ether. Stirring was continued overnight then the solution was carefully poured into $200 \mathrm{~mL}$ of $1 \mathrm{M}$ ammonium chloride solution. The organic layer was decanted and the aqueous layer was extracted with dichloromethane. The combination of the organic layers was evaporated, then 
toluene and a catalytic amount of $p$-toluenesulfonic acid were added. The flask was fitted with a Dean-Stark apparatus and the solution was refluxed for approximatively 45 min (monitored by TLC). After cooling, triethylamine $(2 \mathrm{~mL})$ and phthalic anhydride $(1.48 \mathrm{~g}, 10 \mathrm{mmol})$ were added, then the solution was refluxed (Dean-Stark apparatus) for 2 days. The solution was cooled, then was concentrated under reduced pressure. The residue was recrystallized from ethanol to afford pure phthalimides $\mathbf{3 a}, \mathbf{b}$.

\section{2-[2-(1-methylethenyl)phenyl]phthalimide (3a)}

Yield 91\%; mp $139^{\circ} \mathrm{C}$; IR: $1710(\mathrm{C}=\mathrm{O}) \mathrm{cm}^{-1} ;{ }^{1} \mathrm{H}$ NMR $\left(\mathrm{CDCl}_{3}\right): \delta 1.97-2.10\left(\mathrm{~m}, 3 \mathrm{H}, \mathrm{CH}_{3}\right)$, 4.73-4.82 (m, $\left.1 \mathrm{H},=\mathrm{CH}_{2}\right), 4.92-5.01\left(\mathrm{~m}, 1 \mathrm{H},=\mathrm{CH}_{2}\right), 7.19-7.49\left(\mathrm{~m}, 4 \mathrm{H}, \mathrm{H}_{\text {arom }}\right), 7.70-7.82(\mathrm{~m}$, $2 \mathrm{H}, \mathrm{H}_{\text {arom }}$ ), 7.85-7.99 (m, 2H, $\mathrm{H}_{\text {arom }}$ ); Anal. Calcd. For $\mathrm{C}_{17} \mathrm{H}_{13} \mathrm{NO}_{2}: \mathrm{C}, 77.55 ; \mathrm{H}, 4.98 ; \mathrm{N}, 5.32$. Found: C, 77.32; H, 5.01; N, 5.32.

\section{2-[2-(1-phenylethenyl)phenyl]phthalimide (3b)}

Yield 97\%; mp $193{ }^{\circ} \mathrm{C}$; IR: $1723(\mathrm{C}=\mathrm{O}) \mathrm{cm}^{-1} ;{ }^{1} \mathrm{H}$ NMR $\left(\mathrm{CDCl}_{3}\right): \delta 5.41(\mathrm{~d}, \mathrm{~J}=1 \mathrm{~Hz}, 1 \mathrm{H}$, $\left.=\mathrm{CH}_{2}\right), 4.92-5.01\left(\mathrm{~d}, \mathrm{~J}=1 \mathrm{~Hz}, 1 \mathrm{H},=\mathrm{CH}_{2}\right), 6.80-7.73\left(\mathrm{~m}, 13 \mathrm{H}, \mathrm{H}_{\text {arom }}\right)$; Anal. Calcd. for $\mathrm{C}_{22} \mathrm{H}_{15} \mathrm{NO}_{2}$ : C, 81.21; H, 4.65; N, 4.31. Found: C, 80.94; H, 4.60; N, 4.33.

\section{Synthesis of hydroxylactams 4a,b}

General procedure: To a mixture of phthalimide 3a,b $(4 \mathrm{mmol})$ in dry methanol $(40 \mathrm{~mL})$ at $10^{\circ} \mathrm{C}$ were added sodium borohydride by portions and 5 drops of ethanolic hydrochloric acid solution (prepared from 9 drops of concentrated hydrochloric acid in $15 \mathrm{~mL}$ of ethanol) at regular intervals (10 minutes) until the reaction was complete (controlled by TLC). The excess of sodium borohydride was decomposed by careful addition of diluted hydrochloric acid. Sodium hydrogen carbonate was added and the solvent was evaporated. The residue was triturated with water and the hydroxylactam $\mathbf{4 a , b}$ was separated by filtration, washed with water, dried and recrystallized from ethanol.

\section{2,3-Dihydro-3-hydroxy-2-[2-(1-methylethenyl)phenyl]-1H-isoindol-1-one (4a)}

Yield 97\%; mp 230 ${ }^{\circ}$; IR: $3260(\mathrm{OH}), 1661(\mathrm{C}=\mathrm{O}) \mathrm{cm}^{-1} ;{ }^{1} \mathrm{H}$ NMR $\left(\mathrm{CDCl}_{3}\right): \delta 1.99(\mathrm{~s}, 3 \mathrm{H}$, $\left.\mathrm{CH}_{3}\right), 2.67(\mathrm{~s}, 1 \mathrm{H}, \mathrm{OH}), 4.97-5.02\left(\mathrm{~m}, 1 \mathrm{H},=\mathrm{CH}_{2}\right), 5.04-5.10\left(\mathrm{~m}, 1 \mathrm{H},=\mathrm{CH}_{2}\right), 6.14(\mathrm{~s}, 1 \mathrm{H}, \mathrm{CH})$, 7.20-7.67 (m, 7H, $\left.\mathrm{H}_{\text {arom }}\right), 7.86\left(\mathrm{~d}, \mathrm{~J}=7 \mathrm{~Hz}, 1 \mathrm{H}, \mathrm{H}_{\text {arom }}\right)$; Anal. Calcd. for $\mathrm{C}_{17} \mathrm{H}_{15} \mathrm{NO}_{2}: \mathrm{C}, 76.96$; H, 5.70; N, 5.28. Found: C, 76.63; H, 5.70; N, 5.24.

\section{2,3-Dihydro-3-hydroxy-2-[2-(1-phenylethenyl)phenyl]-1H-isoindol-1-one (4b)}

Yield 96\%; mp $113^{\circ} \mathrm{C}$; IR: $3395(\mathrm{OH}), 1696(\mathrm{C}=\mathrm{O}) \mathrm{cm}^{-1} ;{ }^{1} \mathrm{H}$ NMR $\left(\mathrm{CDCl}_{3}\right): \delta 2.88(\mathrm{~d}, \mathrm{~J}=10$ $\mathrm{Hz}, 1 \mathrm{H}, \mathrm{OH}), 5.39\left(\mathrm{~s}, 1 \mathrm{H},=\mathrm{CH}_{2}\right), 5.52\left(\mathrm{~s}, 1 \mathrm{H},=\mathrm{CH}_{2}\right), 5.69(\mathrm{~d}, \mathrm{~J}=10 \mathrm{~Hz}, 1 \mathrm{H}, \mathrm{CH}), 6.87-7.65$ 
(m, $13 \mathrm{H}, \mathrm{H}_{\text {arom }}$ ); Anal. Calcd. for $\mathrm{C}_{22} \mathrm{H}_{17} \mathrm{NO}_{2}: \mathrm{C}, 80.71 ; \mathrm{H}, 5.23 ; \mathrm{N}, 4.28$. Found: $\mathrm{C}, 80.65 ; \mathrm{H}$, $5.17 ; \mathrm{N}, 4.30$.

\section{Synthesis of isoindoloquinolines 12a,b}

First step: To a solution of imide 3a,b $(10 \mathrm{mmol})$ in dry dichloromethane $(20 \mathrm{~mL})$ was added a solution of methylmagnesium iodide $(0.5 \mathrm{M}$ in ether, $60 \mathrm{~mL}, 30 \mathrm{mmoles})$. The resulting mixture was stirred for 2 hours at room temperature, then poured into $200 \mathrm{~mL}$ of $1 \mathrm{M}$ ammonium chloride solution and extracted with dichloromethane. The organic layer was dried over magnesium sulfate and the solvent was removed under reduced pressure. Methylhydroxylactams $\mathbf{5 a}, \mathbf{b}$ were contaminated by their corresponding enamides $\mathbf{6 a , b}$.

Second step: A mixture of 5a-6a (or 5b-6b), a catalytic amount of $p$-toluenesulfonic acid and toluene were refluxed in a flask fitted with a Dean- Stark apparatus for 4 hours. The solution was cooled, washed with an aqueous solution of sodium hydrogen carbonate, dried over magnesium sulfate, then was concentrated under reduced pressure.

\section{5,6a-Dimethylisoindolo[2,1-a]quinolin-11(6aH)-one (12a)}

Yield 95\% (oil); ${ }^{1} \mathrm{H}$ NMR $\left(\mathrm{CDCl}_{3}\right.$ ): $\delta 1.49$ (s, 3H, $\mathrm{CH}_{3}$ ), 2.05 (d, J = $1 \mathrm{~Hz}, 3 \mathrm{H}, \mathrm{CH}_{3}$ ), 6.00 (d, $\left.\mathrm{J}=1 \mathrm{~Hz}, 1 \mathrm{H}, \mathrm{H}_{6}\right), 7.09-7.67\left(\mathrm{~m}, 6 \mathrm{H}, \mathrm{H}_{\text {arom }}\right), 7.90\left(\mathrm{~d}, \mathrm{~J}=8 \mathrm{~Hz}, 1 \mathrm{H}, \mathrm{H}_{\text {arom }}\right), 7.99$ (d, J = $8 \mathrm{~Hz}$,

1H, $\left.\mathrm{H}_{\text {arom }}\right) ;{ }^{13} \mathrm{C}$ NMR: $\delta 18.7\left(\mathrm{CH}_{3}\right), 26.6\left(\mathrm{CH}_{3}\right), 62.9(\mathrm{C}), 120.9(\mathrm{CH}), 122.5(\mathrm{CH}), 124.0$ $(\mathrm{CH}), 124.3(\mathrm{CH}), 124.7(\mathrm{CH}), 127.6(\mathrm{C}), 127.9(\mathrm{CH}), 128.1(\mathrm{CH}), 128.4(\mathrm{CH}), 130.4(\mathrm{C})$, $130.8(\mathrm{C}), 132.5(\mathrm{CH}), 132.8(\mathrm{C}), 148.7(\mathrm{C}), 166.3(\mathrm{CO})$.

\section{5,6a-Methyl-5-phenylisoindolo[2,1-a]quinolin-11(6aH)-one (12b)}

Yield 93\%; mp 229 ${ }^{\circ} \mathrm{C}$ (ethanol); IR: $1699(\mathrm{C}=\mathrm{O}) \mathrm{cm}^{-1} ;{ }^{1} \mathrm{H}$ NMR $\left(\mathrm{CDCl}_{3}\right): \delta 1.61(\mathrm{~s}, 3 \mathrm{H}$, $\left.\mathrm{CH}_{3}\right), 6.15$ (s, 1H, $\left.\mathrm{H}_{6}\right), 7.07-7.66\left(\mathrm{~m}, 11 \mathrm{H}, \mathrm{H}_{\text {arom }}\right), 7.93$ (d, J = $\left.8 \mathrm{~Hz}, 1 \mathrm{H}, \mathrm{H}_{\text {arom }}\right), 8.06$ (d, J = 8 $\left.\mathrm{Hz}, 1 \mathrm{H}, \mathrm{H}_{\text {arom }}\right){ }^{13} \mathrm{C}$ NMR: $\delta 26.1\left(\mathrm{CH}_{3}\right), 63.0(\mathrm{C}), 121.0(\mathrm{CH}), 122.8(\mathrm{CH}), 124.4(\mathrm{CH}), 124.5$ $(\mathrm{CH}), 126.7(\mathrm{CH}), 127.0(\mathrm{C}), 127.8(\mathrm{CH}), 128.2(2 \mathrm{CH}), 128.3(\mathrm{CH}), 128.7(2 \mathrm{CH}), 128.8$ (CH), $129.2(\mathrm{CH}), 130.9(\mathrm{C}), 132.7(\mathrm{CH}), 133.2(\mathrm{C}), 137.6(\mathrm{C}), 138.3(\mathrm{C}), 148.4(\mathrm{C}), 166.2$ (CO); Anal. Calcd. For $\mathrm{C}_{23} \mathrm{H}_{17} \mathrm{NO}$ C, 85.42; H, 5.30; N, 4.33. Found: C, 85.17; H, 5.23; N, 4.19.

\section{5-Methylisoindolo[2,1-a]quinolin-11(5H)-one (13a)}

A mixture of $4 \mathbf{a}(2.63 \mathrm{~g}, 10 \mathrm{mmol})$, a catalytic amount of $p$-toluenesulfonic acid and toluene were refluxed in a flask fitted with a Dean-Stark apparatus for $30 \mathrm{~min}$. The solution was cooled, washed with an aqueous solution of sodium hydrogen carbonate, dried over magnesium sulfate, then was concentrated under reduced pressure. The residue was 
recrystallized from ethanol. Yield 93\%; mp $149^{\circ} \mathrm{C}$; IR: $1679(\mathrm{C}=\mathrm{O}) \mathrm{cm}^{-1} ;{ }^{1} \mathrm{H} \mathrm{NMR}\left(\mathrm{CDCl}_{3}\right)$ : $\delta 1.44\left(\mathrm{~d}, \mathrm{~J}=7 \mathrm{~Hz}, 3 \mathrm{H}, \mathrm{CH}_{3}\right)$,

$3.82\left(\mathrm{qd}, \mathrm{J}=7\right.$ and $\left.5 \mathrm{~Hz}, 1 \mathrm{H}, \mathrm{H}_{5}\right), 5.95\left(\mathrm{~d}, \mathrm{~J}=5 \mathrm{~Hz}, 1 \mathrm{H}, \mathrm{H}_{6}\right), 7.07-7.72\left(\mathrm{~m}, 6 \mathrm{H}, \mathrm{H}_{\text {arom }}\right), 7.87$ $\left(\mathrm{d}, \mathrm{J}=8 \mathrm{~Hz}, 1 \mathrm{H}, \mathrm{H}_{\text {arom}}\right), 8.98\left(\mathrm{~d}, \mathrm{~J}=8 \mathrm{~Hz}, 1 \mathrm{H}, \mathrm{H}_{\text {arom }}\right) ;{ }^{13} \mathrm{C} \mathrm{NMR}: \delta 25.3\left(\mathrm{CH}_{3}\right), 32.2(\mathrm{CH})$, 109.4 (CH), $117.5(\mathrm{CH}), 119.3(\mathrm{CH}), 123.3(\mathrm{CH}), 124.8(\mathrm{CH}), 127.4(\mathrm{CH}), 127.9(\mathrm{C}), 128.4$ $(\mathrm{CH}), 129.1(\mathrm{CH}), 130.2(\mathrm{C}), 132.0(\mathrm{CH}), 132.6(\mathrm{C}), 133.6(\mathrm{C}), 134.2(\mathrm{C}), 165.2(\mathrm{CO})$; Anal. Calcd. for $\mathrm{C}_{17} \mathrm{H}_{13} \mathrm{NO}$ : C, 82.57; H, 5.30; N, 5.66. Found: C, 82.20; H, 5.25; N, 5.52.

\section{5-Phenylisoindolo[2,1-a]quinolin-11(5H)-one (13b)}

A solution of hydroxylactam $\mathbf{4 b}(10 \mathrm{mmol})$, dichloromethane $(50 \mathrm{~mL})$, trifluoroacetic acid $(5$ $\mathrm{mL}$ ) was stirred for 3 days at room temperature. After concentration under reduced pressure, the residue was dissolved into dichloromethane. The solution was washed with a solution of sodium hydrogen carbonate, dried, then concentrated. The residue was recrystallized from ethanol to afford pure 13b. Yield 91\%; mp $173^{\circ} \mathrm{C}$; IR: $1699(\mathrm{C}=\mathrm{O}) \mathrm{cm}^{-1} ;{ }^{1} \mathrm{H} \mathrm{NMR}\left(\mathrm{CDCl}_{3}\right)$ : $\delta$ $4.97\left(\mathrm{~d}, \mathrm{~J}=4 \mathrm{~Hz}, 1 \mathrm{H}, \mathrm{H}_{5}\right), 6.02\left(\mathrm{~d}, \mathrm{~J}=4 \mathrm{~Hz}, 1 \mathrm{H}, \mathrm{H}_{6}\right), 6.86-8.00\left(\mathrm{~m}, 12 \mathrm{H}, \mathrm{H}_{\text {arom }}\right), 9.05$ (d, J = 8

$\left.\mathrm{Hz}, 1 \mathrm{H}, \mathrm{H}_{\text {arom }}\right) ;{ }^{13} \mathrm{C}$ NMR: $\delta 43.8(\mathrm{CH}), 107.4(\mathrm{CH}), 117.2(\mathrm{CH}), 119.3(\mathrm{CH}), 123.0(\mathrm{CH})$, 124.6 (CH), $125.6(\mathrm{C}), 126.7(\mathrm{CH}), 127.4(\mathrm{CH}), 128.1(2 \mathrm{CH}), 128.6(2 \mathrm{CH}), 129.1(\mathrm{CH}), 129.8$ (C), $129.9(\mathrm{CH}), 131.8(\mathrm{C}), 131.9(\mathrm{CH}), 133.4(\mathrm{C}), 134.0(\mathrm{C}), 144.8(\mathrm{C}), 165.0$ (CO); Anal. Calcd. for $\mathrm{C}_{22} \mathrm{H}_{15} \mathrm{NO}$ : C, 85.41; H, 4.89; N, 4.53. Found: C, 85.10; H, 4.85; N, 4.38.

\section{Isoindolo[2,1-a]quinolin-11(5H)-one (13c)}

This compound was prepared from 20 in a similar manner as described for the synthesis of 13a. Yield $99 \%$. The physical data are identical to those reported in the literature ${ }^{7}$

\section{2-(2-benzoylphenyl)phthalimide (16)}

A mixture of phthalic anhydride $(1.48 \mathrm{~g}, 10 \mathrm{mmol})$, o-aminobenzophenone $(1.97 \mathrm{~g}, 10 \mathrm{mmol})$, triethylamine $(2 \mathrm{~mL})$ and toluene was refluxed in a flask fitted with a Dean-Stark apparatus for 2 days. The solution was cooled, then was concentrated under reduced pressure. The residue was recrystallized from ethanol to afford pure phthalimide 16 . Yield $93 \%$; $\mathrm{mp} 200^{\circ} \mathrm{C}$; IR: $1709(\mathrm{C}=\mathrm{O}), 1665(\mathrm{C}=\mathrm{O}) \mathrm{cm}^{-1} ;{ }^{1} \mathrm{H} \mathrm{NMR}\left(\mathrm{CDCl}_{3}\right)$ : $\delta$ 7.07-7.82 (m, 13H, $\left.\mathrm{H}_{\text {arom }}\right)$; Anal. Calcd. for $\mathrm{C}_{21} \mathrm{H}_{13} \mathrm{NO}_{3}$ : C, 77.06; H, 4.00; N, 4.28. Found: C, 76.82; H, 4.06; N, 4.31.

\section{2,3-Dihydro-3-hydroxy-2-(2-(phenylmethyl)phenyl]-1H-isoindol-1-one (17)}

A mixture of 16 (1.83 g, $10 \mathrm{mmol})$, triethylsilane $(5 \mathrm{~mL}, 30 \mathrm{mmol})$, trifluoroacetic acid (30 $\mathrm{mL}$ ) was stirred for 2 days at room temperature. The solution was concentrated under reduced pressure then methanol was added $(100 \mathrm{~mL})$ and reduction was accomplished with sodium borohydride with the same procedure than for the synthesis of $\mathbf{4 a , b}$. Yield $96 \%$; $\mathrm{mp} 147^{\circ} \mathrm{C}$; 
IR: $3327(\mathrm{OH}), 1682(\mathrm{C}=\mathrm{O}) \mathrm{cm}^{-1} ;{ }^{1} \mathrm{H}$ NMR $\left(\mathrm{CDCl}_{3}\right): \delta 1.64(\mathrm{~s}, 1 \mathrm{H}, \mathrm{CH}), 3.91(\mathrm{~d}, \mathrm{~J}=15 \mathrm{~Hz}$, $\left.1 \mathrm{H}, \mathrm{CH}_{2}\right), 4.06\left(\mathrm{~d}, \mathrm{~J}=15 \mathrm{~Hz}, 1 \mathrm{H}, \mathrm{CH}_{2}\right), 5.63(\mathrm{~s}$ broad, $1 \mathrm{H}, \mathrm{CH}), 6.89-7.61\left(\mathrm{~m}, 12 \mathrm{H}, \mathrm{H}_{\text {arom}}\right)$, 7.80-7.88 (m, $1 \mathrm{H}, \mathrm{H}_{\text {arom }}$ ); Anal. Calcd. for $\mathrm{C}_{21} \mathrm{H}_{17} \mathrm{NO}_{2}$ : C, 79.98; H, 5.43; N, 4.44. Found: C, 79.92; H, 5.44; N, 4.47.

\section{$5 H$-Isoindolo[2,1-a]dibenz[c,f]azepin-14(9bH)-one (18)}

A solution of $17(1.85 \mathrm{~g}, 10 \mathrm{mmol})$ in trifluoroacetic acid $(30 \mathrm{~mL})$ was stirred overnight at room temperature. The solution was concentrated under reduced pressure then the residue was recrystallized from ethanol to afford pure 18. Yield 95\%; mp $183^{\circ} \mathrm{C}$; IR: $1698(\mathrm{C}=\mathrm{O}) \mathrm{cm}^{-1} ;{ }^{1} \mathrm{H}$ NMR $\left(\mathrm{CDCl}_{3}\right): \delta 3.54\left(\mathrm{~d}, \mathrm{~J}=14 \mathrm{~Hz}, 1 \mathrm{H}, \mathrm{H}_{5}\right), 4.22\left(\mathrm{~d}, \mathrm{~J}=14 \mathrm{~Hz}, 1 \mathrm{H}, \mathrm{H}_{5}\right), 6.06\left(\mathrm{~s}, 1 \mathrm{H}, \mathrm{H}_{9 \mathrm{a}}\right)$, 6.93-7.72 (m, 11H, $\left.\mathrm{H}_{\text {arom}}\right), 7.98\left(\mathrm{~d}, \mathrm{~J}=7 \mathrm{~Hz}, 1 \mathrm{H}, \mathrm{H}_{\text {arom }}\right) ;{ }^{13} \mathrm{C} \mathrm{NMR}: \delta 39.0\left(\mathrm{CH}_{2}\right), 64.5(\mathrm{CH})$, $122.9(\mathrm{CH}), 124.2(\mathrm{CH}), 127.2(\mathrm{CH}), 127.8(2 \mathrm{CH}), 128.0(\mathrm{CH}), 128.1(\mathrm{CH}), 128.4(\mathrm{CH})$, 128.5

(2CH), $130.0(\mathrm{CH}), 131.4(\mathrm{C}), 132.2(\mathrm{CH}), 134.4(\mathrm{C}), 136.4(\mathrm{C}), 137.1(\mathrm{C}), 138.9(\mathrm{C}), 144.7$ (C), 168.0 (CO); Anal. Calcd. for $\mathrm{C}_{21} \mathrm{H}_{15} \mathrm{NO}$ : C, 84.82; H, 5.08; N, 4.71. Found: C, 84.94; H, $4.97 ; \mathrm{N}, 4.68$.

\section{REFERENCES AND NOTES}

1 Othman, M.; Pigeon, P.; Decroix, B., Tetrahedron, 1997, 53, 2495-2504.

2 Pigeon, P.; Decroix, B., Tetrahedron Lett., 1997, 38, 1041-1042.

3 Pigeon, P.; Decroix, B., Tetrahedron Lett., 1997, 38, 2985-2988.

4 Speckamp, W.N.; Hiemstra, H., Tetrahedron, 1985, 41, 4367-4416.

5 Hiemstra, H; Speckamp, W.N., Comprehensive Organic Synthesis: Trost, B.M.; Fleming, I. Eds Pergamon, Oxford, 1991, vol 2, pp 1047-1082.

6 Ishihara, Y.; Kiyota, Y.; Goto, G., Chem. Pharm. Bull., 1990, 38, 3024-3030.

7 Abe, Y.; Ohsawa, A.; Igeta, H., Heterocycles, 1982, 19, 49-51.

8 Alkhathlan, H.Z., J. Chem. Research (S), 1992, 260-261.

9 Pigeon, P.; Decroix, B., Bull. Soc. Chim. Fr., 1997, 134, 153-157.

10 Pigeon, P.; Decroix, B., J. Heterocyclic Chem., 1996, 33, 129-135. 